\title{
Developing Learning Scenarios to Foster Children's Handwriting Skills with the Help of Social Robots
}

Conference Paper · March 2017

DOI: $10.1145 / 3029798.3034818$

CITATIONS

0

3 authors:

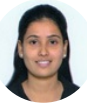

Shruti Chandra

École Polytechnique Fédérale de Lausanne

4 PUBLICATIONS 6 CITATIONS

SEE PROFILE

\section{Ana Paiva}

Instituto Superior Técnico, Inesc-ID

406 PUBLICATIONS $\quad \mathbf{4 , 7 7 4}$ CITATIONS

SEE PROFILE
READS

43
Pierre Dillenbourg

École Polytechnique Fédérale de Lausanne 357 PUBLICATIONS 9,816 CITATIONS

SEE PROFILE

Some of the authors of this publication are also working on these related projects:

All content following this page was uploaded by Pierre Dillenbourg on 16 March 2017. 


\title{
Developing Learning Scenarios to Foster Children's Handwriting Skills with the Help of Social Robots
}

\author{
Shruti Chandra ${ }^{\dagger, \star}$, Pierre Dillenbourg ${ }^{\dagger}$ and Ana Paiva* \\ $\dagger^{\dagger} \mathrm{CHILI}$, École Polytechnique Fédérale de Lausanne, Switzerland \\ *INESC-ID \& Instituto Superior Técnico, Universidade de Lisboa, Portugal \\ shruti.chandra@tecnico.ulisboa.pt, pierre.dillenbourg@epfl.ch, ana.paiva@inesc-id.pt
}

\begin{abstract}
Social robots are being used to create better educational scenarios, boosting children's motivation and engagement. The focus of the research is to explore new ways to support children in acquisition of their handwriting skills with the help of a social robot. With this perspective, three studies are discussed to investigate aspects related to the learning modes of child-robot interaction, children's impression of a social robot and classification of children's common handwriting difficulties.
\end{abstract}

\section{Keywords}

Social Robotics; Child-Robot Interaction; Learning methods

\section{INTRODUCTION}

Handwriting difficulties negatively affect children's academic performance [4] and their self-esteem [9]. The process of handwriting is a complex blend of motor and cognition skills. Ineffective motor skills are difficult to correct once they are acquired, therefore special attention should be given to preschoolers from the very beginning [11]. In the past few years, social robots are being introduced into educational context seeking different ways of fostering children's learning experience. For example, Kanda et al. [7], used Robovie robot, as an English peer-tutor for Japanese students. The results showed the robot encouraged some of the students to improve their English and formed relationships with them.

The current research focuses on a long term question of how a social robot can help children to acquire handwriting skills. With the aim to create better learning scenarios, we investigate the following aspects: 1) Modes of Interaction - to determine which modes of child-robot interaction better support acquisition of hand-writing skills; 2) Children's impressions of a social robot - to understand how children perceive the robot's behavior and it's abilities through the modes of interactions; 3) Handling Writing difficulties - to explore common handwriting difficulties which differ from a child to child according to their age, cognitive and motor skills.

Permission to make digital or hard copies of all or part of this work for personal or classroom use is granted without fee provided that copies are not made or distributed for profit or commercial advantage and that copies bear this notice and the full citation on the first page. Copyrights for components of this work owned by others than ACM must be honored. Abstracting with credit is permitted. To copy otherwise, or republish, to post on servers or to redistribute to lists, requires prior specific permission and/or a fee. Request permissions from permissions@ acm.org.

HRI'17 Companion March 06-09, 2017, Vienna, Austria

(C) 2017 ACM. ISBN 978-1-4503-4885-0/17/03.

DOI: http://dx.doi.org/10.1145/3029798.3034818

\section{RESEARCH}

To explore which modes of interactions are more effective for children, we first conducted a child-child study (study\#1) in the age group 5-6 years, with a human facilitator without involving a robot. In this study, we examined two peerassisted learning methods: the Peer-tutoring (PT) and the Peer-learning (PL) method which are supposed to be effective in schools [1]. The goals of the study were: 1 ) to contrast the child-child interaction in both learning methods. 2) to create a database of children's handwritten letters and handwriting issues. The scenario comprised a pair of children performing a collaborative writing activity (on paper and a tactile device) in the presence of a human facilitator. In the "PT condition" one child acted as a teacher and the other as a learner, while in the "PL condition" both the children were learners without the attribution of any specific role. In the PT condition, the teacher-child provided the corrective feedback (verbal/non-verbal) on the performance of the learner child; while in the PL condition both the children provided corrective feedback on each other's performance as both acted as a learner as well as a teacher. The role of the human facilitator was to provide the instructions during the activity. The results suggests that in terms of communication modalities such as verbal responses and gestures, the PL condition was richer than the PT condition. However, in terms of children's activeness (excitement), the PT was richer than the PL condition. Based on the database collected from the handwritten letters, a taxonomy of handwriting issues was also created, matching many of the issues previously suggested by Graham et al. [5] such as letter formation, alignment of letters.

The results of the study\#1 indicated the effectiveness of both learning methods in different aspects, however, we found children were immature in providing corrective feedback on their peers performance. Thus, we conducted the second study (study\#2) with children in the age group 6-8 years, replicating the protocol but involving a social robot besides a human facilitator. In order to explore the impact of introducing the robot facilitator, the study was carried out under three conditions: 1) a pair of children with a human facilitator, performing a collaborative writing activity based on the PT method; 2) identical to the condition 1, but replacing the human facilitator to the robot facilitator and ; 3) a pair of children with the robot facilitator performing the activity based on the PL method. The children were assigned a role of a teacher or a learner according to the learning method used in each condition. The role of the facilitators was to provide the interaction flow during the 
activity. A Wizard-of-Oz procedure was used to control the robot's behavior. The goals of the study were to explore: the children's responsibility in the presence of both the facilitators; which learning method would lead to more children's corrective feedback to their peers and self-disclosure to the facilitators. The results showed that the PT situation can lead to significantly more corrective feedback provided by the children to their peers as well as self-disclosure to the robot compared to the $P L$ situation [3]. Other results in the PT situation showed, the tutor-children felt more responsible towards their peers in the presence of the robot contrary to the human facilitator [2]. Results of the children's learning gains suggest there was a significant difference between the pre- and post-test scores in both conditions but no significance difference found between the two learning methods (condition $2 \& 3$ ) and the two facilitators (condition $1 \& 2$ ). Overall, the $P T$ method found to be more effective in terms of children's corrective feedback and self-disclosure.

A significant part of the work in educational robotics deals with robotic tutors, however, recently researchers have used the PT method. For example, Shizuko et al. [10] performed a study with a tele-operated Nao robot where it helped children to learn unknown English words. However, how do children perceive these robots? And how do these perceptions change over long-term interactions? Children's perception of the robots indeed seems to be relevant in child-robot interactions. In fact, Kahn et al. [6] showed majority of children believed the robot has mental states and was considered as a social being. In our third study (study\#3), an autonomous social behavior of a robot was developed to provide an educational scenario for children to improve their handwriting skills. The system relies on the preferred PT method and the goal was to understand how children perceive the robot over time in terms of its behavior and abilities since the aspects such as children's performance, engagement may be altered [8]. Thus, we tested the system by conducting a long-term study with 7-8 years old in a school, the robot exhibited two contrasting competencies: learning and non-learning. The scenario involved a tutor-child correcting handwriting issues of a learner-robot. Instead of generating the random handwriting issues for the robot, we selected three common issues [5] from the previously created taxonomy of handwriting issues and used the algorithm proposed in [12] to generate the deformed letters. In the learning condition, the robot showed learning progression after each interaction while in the non-learning condition it showed consistent performance (it does not learn) through out the study.

Extending the line of work of Shizuko et al. [10], the study\#3 makes two contributions: firstly by developing an autonomous social behavior of the robot, designed to provide a learning scenario where a child learns by teaching the robot; and secondly the understanding of the children's perception of the robot's learning abilities through child self-response questionnaires (partially based on Godspeed questionnaire). In contrast to study \#2, this study provided the robot to play an active role (as a learner) and explores the following research questions: Would the children be able to differentiate the two competencies in the robot; How the robot's competencies affect children's performance, social behavior and tutoring towards it? We expect to answer these questions after completing the data analysis. The findings of the research might bring new elements in order to design better educational scenarios with social robots.

\section{FUTURE WORK}

The next step is to conclude the data analysis of the study\#3 to arrive on the final conclusions. If the children were able to identify robot's competencies, indicating children's awareness of the robot's handwriting issues. We foresee our next study to explore children's performance and their perception towards a robot which is self-aware of its own writing issues. The robot would exhibit self-awareness by commenting on its own writing.

\section{ACKNOWLEDGMENTS}

This work was supported by national funds through Fundação para a Ciência e a Tecnologia (FCT) with reference UID/CEC/500 21/2013 and through project AMIGOS (PTDC/EEISII/ 7174/201 4). The first author acknowledge grants ref. SFRH/BD/519 $35 / 2012$ funded by the FCT. We show our gratitude to all the schools involved in the studies.

\section{REFERENCES}

[1] D. Boud, R. Cohen, and J. Sampson. Peer learning and assessment. Peer Learning in Higher Education: Learning from \& with Each Other, page 67, 2001.

[2] S. Chandra, P. A.Oliveira, S. Lemaignan, P. Sequeira, A. Paiva, and P. Dillenbourg. Can a child feel responsible for another in the presence of a robot in a collaborative learning activity? In Robot and Human Interactive Comm.(RO-MAN), 2015 24th IEEE Int. Symposium on, pages 167-172. IEEE, 2015.

[3] S. Chandra, P. A.Oliveira, S. Lemaignan, P. Sequeira, A. Paiva, and P. Dillenbourg. Children's peer assessment and self-disclosure in the presence of an educational robot. In Robot and Human Interactive Comm.(RO-MAN), 2016 25th IEEE Int. Symposium on, pages 539-544. IEEE, 2016.

[4] C. A. Christensen. The role of orthographic-motor integration in the production of creative and well-structured written text for students in secondary school. Educational Psychology, 25(5):441-453, 2005.

[5] S. Graham, K. R. Harris, L. Mason, B. F.Chorzempa, S. Moran, and B. Saddler. How do primary grade teachers teach handwriting? a national survey. Reading and Writing, 21(1-2):49-69, 2008.

[6] P. H. Kahn Jr, T. Kanda, H. Ishiguro, N. G. Freier, R. L. Severson, B. T. Gill, J. H. Ruckert, and S. Shen. Developmental psychology, (2).

[7] T. Kanda, T. Hirano, D. Eaton, and H. Ishiguro. Interactive robots as social partners and peer tutors for children: A field trial. Human-Computer Interaction, 19(1):61-84, June 2004.

[8] J. Kennedy, P. Baxter, and T. Belpaeme. The robot who tried too hard: Social behaviour of a robot tutor can negatively affect child learning. In Proceedings of the Tenth Annual ACM/IEEE International Conference on Human-Robot Interaction, HRI '15, pages 67-74, New York, NY, USA, 2015. ACM.

[9] T. Malloy-Miller, H. Polatajko, and B. Anstett. Handwriting error patterns of children with mild motor difficulties. Canadian Journal of Occupational Therapy, 62(5):258-267, 1995.

[10] S. Matsuzoe and F. Tanaka. How smartly should robots behave?: Comparative investigation on the learning ability of a care-receiving robot. In 2012 IEEE RO-MAN: The 21st IEEE International Symposium on Robot and Human Interactive Comm., pages 339-344. IEEE, 2012.

[11] J. Medwell and D. Wray. Handwriting-a forgotten language skill? Language and Education, 22(1), 2008.

[12] H. Yin, P. A.Olivera, F. S. Melo, A. Billard, and A. Paiva. Synthesizing robotic handwriting motion by learning from human demonstrations. In Proceedings of International Joint Conference on Artificial Intelligence (IJCAI), 2016. 